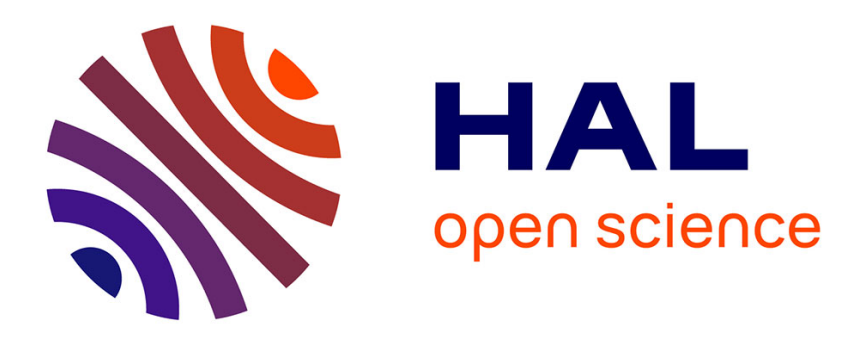

\title{
Simple calculation of sedimentation velocity in concentrated dispersions
}

D. Stauffer, P. Clavin

\section{To cite this version:}

D. Stauffer, P. Clavin. Simple calculation of sedimentation velocity in concentrated dispersions. Journal de Physique Lettres, 1981, 42 (15), pp.353-355. 10.1051/jphyslet:019810042015035300 . jpa00231944

\section{HAL Id: jpa-00231944 https://hal.science/jpa-00231944}

Submitted on 1 Jan 1981

HAL is a multi-disciplinary open access archive for the deposit and dissemination of scientific research documents, whether they are published or not. The documents may come from teaching and research institutions in France or abroad, or from public or private research centers.
L'archive ouverte pluridisciplinaire HAL, est destinée au dépôt et à la diffusion de documents scientifiques de niveau recherche, publiés ou non, émanant des établissements d'enseignement et de recherche français ou étrangers, des laboratoires publics ou privés. 


\title{
LE JOURNAL DE PHYSIQUE-LETTRES
}

\section{Simple calculation of sedimentation velocity in concentrated dispersions}

\author{
D. Stauffer $(*)$ and P. Clavin $\left({ }^{* *}\right)$ \\ Département de Physique des Systèmes Désordonnés, Université de Provence, \\ Centre St-Jérôme, 13397 Marseille Cedex 13, France
}

(Reçu le 16 février 1981, accepté le 9 juin 1981)

Résumé. - La théorie de Batchelor sur la sédimentation des suspensions diluées est généralisée aux concentrations élevées en utilisant la fonction de corrélation d'un système de sphères dures.

Abstract. - Batchelor's theory of sedimentation in dilute dispersions is generalized to the concentrated case, using the pair correlation function of a hard sphere system.

A single sphere of radius $R$ in an incompressible liquid sinks down slowly with velocity

$$
V_{0}=f / 6 \pi \eta R
$$

in a gravitational force field $f$, where $\eta$ is the viscosity. In a dilute irregular dispersion of such spheres, their average sedimentation velocity $V_{s}$ is reduced, according to Batchelor [1], to

$$
V_{\mathrm{s}}=V_{0}(1-6.55 c+\cdots), \quad c \ll 1
$$

where $c=4 \pi R^{3} n / 3$ is the volume fraction of these spheres, $n$ the number of spheres per unit volume. We now try to generalize this result to larger concentrations $c$. (All spheres still have the same radius $R$.)

A seemingly straightforward Monte-Carlo simulation would be to distribute randomly a large number of spheres $k, k=1,2, \ldots, N$, in a fixed volume $V=N / n$ and to approximate the total flow field $v(r)$ by the

${ }^{*}$ ) Present and permanent address : Institut für Theoretische Physik, Cologne University, D-5000 Köln 41, West Germany.

$\left.{ }^{* *}\right)$ Laboratoire de Dynamique et Thermophysique des Fluides, Université de Provence, Centre St-Jérôme, 13397 Marseille Cedex 13, France. superposition $\Sigma_{k} \mathbf{v}_{k}(\mathbf{r})$ of single isolated spheres moving with velocity $V_{\mathrm{s}}$. If a sphere $k$ at the origin falls down with a velocity $\mathbf{V}_{\mathrm{s}}$ its flow field at not too large distances and not too large velocities is $[1,2]$

$$
\begin{aligned}
\mathbf{v}_{k}(\mathbf{r})=\mathbf{V}_{\mathrm{s}}(3 R / 4 r & \left.+R^{3} / 4 r^{3}\right)+ \\
& +\mathbf{n}\left(\mathbf{n} \mathbf{V}_{\mathrm{s}}\right)\left(3 R / 4 r-3 R^{3} / 4 r^{3}\right)
\end{aligned}
$$

where $\mathbf{n}=\mathbf{r} / r$. Thus the velocities $\mathbf{V}_{i}$ of the various spheres $i$ at positions $\mathbf{r}_{i}$ could be determined by a solution of the coupled equations (as suggested by de Gennes [3])

$$
\mathbf{f}=6 \pi \eta R\left(\mathbf{V}_{i}-\Sigma_{k}^{\prime} \mathbf{v}_{k}\left(\mathbf{r}_{i}-\mathbf{r}_{k}\right)\right) .
$$

But it is well known that this method does not work since the sum over all velocities $\mathbf{v}_{\boldsymbol{k}}$ in equation (4) diverges strongly at large distances : Each velocity then decays only as $1 /$ distance, and the sum is threedimensional. Thus a different method has to be used.

"Batchelor renormalization" uses the following trick [1]. If we complete equation (3) by

$$
\mathbf{v}_{k}(\mathbf{r})=\mathbf{V}_{\mathrm{s}} \text { for } r \leqslant R
$$


this flow field describes also the motion of the sphere itself. And the integral of this $\mathbf{v}_{k}(\mathbf{r})$ over the whole volume of the fixed vessel has to vanish exactly since for every volume element which goes down another volume element goes up. (Equations (3), (5) violate this condition; they neglect effects from the walls [4] and from the other spheres. We expect these corrections to be less important for the short-range flow fields employed below.) The same is true for the total velocity field produced by all spheres in the dispersion. Thus for a completely homogeneous distribution of spheres the sum over $k$ in equation (4) vanishes in the average. In reality the distribution of spheres is not random since their volumes cannot overlap : each sphere $i$ centred at $\mathbf{r}_{i}$ is surrounded by a hole of radius $2 R$ centred at $\mathbf{r}_{i}$ where no other sphere $k$ can have its centre $\dot{\mathbf{r}}_{k}$. Assuming a random distribution of spheres outside this hole, the difficult sum in equation (4) thus equals, in the average over all spheres $i$, the negative contribution $-\Delta$ of imaginary spheres distributed in this hole with the same density as the real spheres are distributed outside the hole. A single sphere of radius $R$ in this hole of radius $2 R$ corresponds to a volume fraction $c=1 / 8$ and gives in the average a velocity $\Delta$ at the hole centre, with

$$
\Delta=\int_{r<2 R} \mathbf{v}_{k}(\mathbf{r}) \mathrm{d}^{3} r / \int_{r<2 R} \mathrm{~d}^{3} r=\frac{11}{16} \mathbf{V}_{\mathrm{s}}
$$

from equations (3), (5). For small $c$, there is either one or no sphere in the hole and its average contribution to the velocity is $\Delta=\frac{11}{16} V_{\mathrm{s}} .8 c=\frac{11}{2} c V_{\mathrm{s}}$. Thus equation (4) is replaced in this limit by

$$
\mathbf{f}=6 \pi \eta R\left(V_{\mathrm{s}}+\Delta\right)=6 \pi \eta R V_{\mathrm{s}}\left(1+\frac{11}{2} c\right)
$$

giving

$$
V_{\mathrm{s}}=V_{0} /(1+5.5 c)=V_{0}(1-5.5 c+\cdots) .
$$

The difference between the factor 5.5 here and the coefficient 6.55 of equation (2) arises from smaller effects neglected in this simple presentation of Batchelor's theory (and in our following calculations). The theoretical result (2) is in good agreement with some experiments [4].

As a first approximation for higher concentrations $c$ we now employ the same methods and simply allow more than one sphere in the hole. A small number $N=1,2,3,4,5$ of spheres is now distributed randomly (except for excluded volume effects) in the hole of radius $2 R$. We still neglect, in the spirit of a mean field approximation [8], the deformation of the velocity field of one sphere due to the presence of the other spheres. (Famularo and Happel [4] took into account the deformation due to the vessel walls, as calculated for a single sphere falling down in a cylinder. We doubt the validity of this approach for a suspension of many spheres where instead the other spheres play the role of walls and screen [7] the velocity field for distances much larger than the average distance between spheres. Perhaps the computational techniques of Ganatos et al. [5] allow a quantitative estimate of this screening effect but we have not undertaken such a calculation.)

The additional contributions calculated by Batchelor and neglected in the presentation above are relatively small $(6.55-5.5 \ll 5.5)$ for dilute solutions. We hope they remain small for the concentrated case and neglect them here completely in a first step. Just as Batchelor we also assume a stationary suspension of identical spheres distributed homogeneously in the vessel; experimentally this situation corresponds to a continuous influx of new spheres at the upper surface. Also, we neglect as does Batchelor that hydrodynamic forces may produce deviations, changing perhaps in time, from the rather random distribution assumed in our calculations.

By averaging over $10^{4}$ configurations we find the sedimentation velocity $V_{\mathrm{s}}$ in this approximation. The velocity correction $\Delta$ is now the sum over $N$ different fields at the origin, as given by equations (3), (5). We put randomly one sphere centre into the hole, and then put another sphere into the same hole by using different random numbers. When this last sphere overlapped with the previous sphere it is thrown away, and a new position is searched randomly. This simple process was repeated until $N$ spheres had their centre in the hole of radius $2 R$, without overlapping with each other.

If $\Delta_{0}$ is the average velocity produced at the hole centre by the $N$ spheres if they all fall down with velocity $V_{0}$, then $\Delta=\Delta_{0} V_{\mathrm{s}} / V_{0}$ is the velocity correction if they fall with speed $V_{\mathrm{s}}$. Thus equation $(6 b)$ is now $f=6 \pi \eta R\left(V_{\mathrm{s}}+\Delta_{0} V_{\mathrm{s}} / V_{0}\right)$ and gives

$$
V_{\mathrm{s}}=V_{0} /\left(1+\Delta_{0} / V_{0}\right) \text {. }
$$

The dots in figure 1 give our results from the MonteCarlo evaluation of $\Delta_{0} / V_{0}$; they lie significantly but only slightly above the Batchelor curve $V_{\mathrm{s}}=V_{\mathrm{o}} /\left(1+\frac{11}{2} c\right)$, shown there as a dashed line. (Though $N$ is fluctuating for a given $c$ we relate it to $c$ simply by $c=N / 8$.)

This crude Monte-Carlo simulation neglects that particularly at high concentrations the many other spheres in the dispersion push the $N$ spheres closer to the centre and that slightly outside the whole of radius $2 R$ the density of spheres is higher than the average. Assuming again the positions of the spheres in the (dilute) dispersion to be well approximated by the hard-sphere correlation function, we now employ a better method by using the published [6] pair correlation function $g(r)$, normalized to $g(r \rightarrow \infty) \rightarrow 1$, of a hard sphere system at the same 


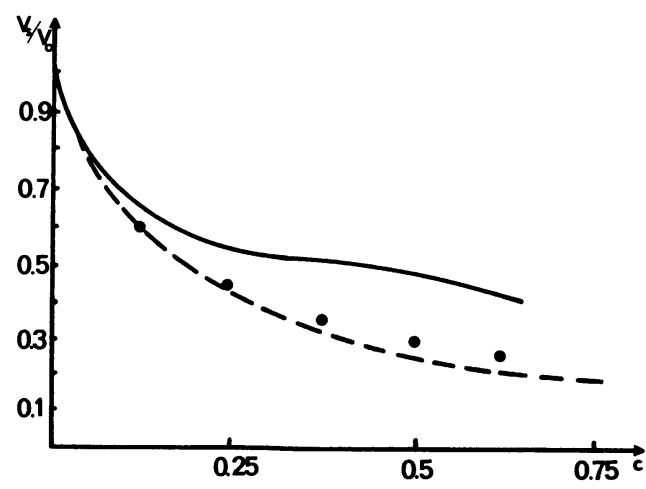

Fig. 1. - Variation of the sedimentation velocity $V_{\mathrm{s}}$, in units of $V_{0}=f / 6 \pi \eta R$, as a function of volume fraction $c$. The dashed line is the Batchelor limit in the form $V_{\mathrm{s}} V_{0}=1 /(1+5.5 c)$, the solid line our better approximation of equation (10). Our other approximation, the simple Monte-Carlo simulation, is shown by the dots, with a statistical error smaller than the symbol size.

volume fraction as the concentration in our suspensions. Then the average velocity $-\Delta$ produced by all the other spheres in the vessel (we no longer restrict ourselves to a small hole) at the centre of one sphere is

$-\Delta=n \int \mathrm{d}^{3} r \mathbf{v}_{k}(\mathbf{r}) g(r)=n \int \mathrm{d}^{3} r \mathbf{v}_{k}(\mathbf{r})(g(r)-1)$

where again $n$ is the number of spheres per unit volume; in the last equality we used again the Batchelor trick that the integral over all velocities is zero. The approximation $g(r)=0$ for $r<2 R$ and $=1$ otherwise recovers equation (6). Using for $r>2 R$ instead the molecular dynamics values tabulated in reference [6], we get

$$
\begin{aligned}
\Delta / V_{\mathrm{s}}=c\left(\frac{11}{2}-3 \int_{2 R}^{\infty}(g(r)-1) r \mathrm{~d} r\right) \simeq & \\
& \simeq c\left(\frac{11}{2}-12 c+11 c^{2}\right) .
\end{aligned}
$$

(The last equation is an empirical fit of moderate accuracy on the integrals calculated from the tables of $g(r)$, for $c$ between 0.2 and 0.5 , and not a systematic expansion in powers of $c$ exact for small $c$.) Equilibrium between gravitational force and friction now gives $f=6 \pi \eta R\left(V_{\mathrm{s}}+\Delta\right)$ or

$$
V_{\mathrm{s}}=V_{0} /\left(1+5.5 c-12 c^{2}+11 c^{3}\right) .
$$

This result is shown as a solid line in our figure and lies appreciably above the previous prediction. Since we still neglect the deformation of the velocity fields and simply used equations (3), (5) we presumably overestimate the influence of each sphere on its more distant neighbours, where screening could become important. Thus the true result is likely between our two results (if the spheres are distributed as in a hard sphere system).

In a real suspension, the distribution of spheres may deviate from that of hard spheres, due to their hydrodynamic interactions, and may change in the course of time [1]. If strong clumping occurs the sedimentation velocity might even be higher than $V_{0}$, even in dilute dispersions [4].

In summary, our figure gives a simple overall approximation and not a systematic perturbation theory valid exactly in certain limits.

Acknowledgments. - We thank E. Guyon and R. Blanc for introducing us into the field of dispersions, F. W. Hehl and P. Pelce for helpful discussions and comments, and R. Blanc for a critical reading of the manuscript.

\section{References}

[1] BATCheLOR, G. K., J. Fluid Mech. 52 (1972) 245.

[2] LANDAU, L. D. and LifCHITZ, E., Mécanique des Fluides (Editions Mir, Moscow) 1971.

[3] De Gennes, P. G., J. Physique 40 (1979) 783.

[4] Famularo, J. and HaPPel, J., Am. Inst. Chem. Eng. J. 11 (1965) 981.
[5] Ganatos, P., Pfeffer, R. and Weinbaum, S., J. Fluid Mech. 84 (1978) 79

[6] Alder, B. J. and Hecht, C. E., J. Chem. Phys. 50 (1969) 2032.

[7] Childress, S., J. Chem. Phys. 56 (1972) 2527.

[8] Kirkpatrick, S., Rev. Mod. Phys. 45 (1973) 574. 